\title{
Können Protronenpumpenhemmer gastrointestinale Blutungen verhindern?
}

Fragestellung: Patienten die wegen eines vaskulären Ereignisses Antithrombotika bekommen und gleichzeitig nicht steroidale Antirheumatika einnehmen, haben ein erhöhtes Blutungsrisiko. Bisher ist nicht sicher, ob dieses Risiko durch die Einnahme von Protronenpumpenhemmern reduziert werden kann.

Hintergrund: Patienten, die einen Herzinfarkt oder Schlaganfall erlitten haben, werden üblicherweise mit Thrombozytenfunktionshemmern behandelt. Wenn sie Vorhofflimmern haben, kommen noch Antikoagulanzien hinzu. Diese Therapie per se hat bereits ein erhöhtes Blutungsrisiko. Dies wird durch die gleichzeitige Einnahme nicht steroidaler Antirheumatika erhöht, wobei dann bevorzugt gastrointestinale Blutungen auftreten. Es stellt sich hier die Frage, ob Patienten, die gleichzeitig Antithrombotika und nicht steroidale Antirheumatika einnehmen, auch routinemäßig Protronenpumpenhemmer erhalten sollten.

Patienten und Methodik: Es handelt sich um eine Analyse des dänischen nationalen Krankenregisters in dem Daten aller Krankenhäuser zwischen 1997

Schjerning Olsen AM, Lindhardsen J, Gislason GH et al. Impact of proton pump inhibitor treatment on gastrointestinal bleeding associated with non-steroidal anti-inflammatory drug use among post-myocardial infarction patients taking antithrombotics: nationwide study. BMJ 2015;351:h5096 und 2011 erfasst sind. Eingeschlossen wurden Patienten im Alter über 30 Jahren mit einem ersten Herzinfarkt, der über einen Zeitraum von mindestens 30 Tagen nach der Krankenhausentlassung überlebt wurde. Erfasst wurden die Einnahme von antithrombotischer Therapie, die
Einnahme nicht steroidaler Antirheumatika und die Einnahme von Protronenpumpenhemmern. Der primäre Endpunkt war die Häufigkeit gastrointestinaler Blutungen.

Ergebnisse: In die Studie wurden 82.955 Patienten nach einem Myokardinfarkt eingeschlossen. Das mittlere Alter betrug 67 Jahre. Von den Studienteilnehmern waren $64 \%$ Männer. Alle Patienten wurden entweder mit einem oder mit zwei Thrombozytenfunktionshemmern behandelt. Von der Gesamtpopulation von 82.955 Patienten hatten 68.044 weder ein nicht steroidales Antirheumatikum noch einen Protronenpumpenhemmer eingenommen. 2.006 Patienten erhielten ein nicht steroidales Antirheumatikum, 12.334 einen Protronenpumpenhemmer und 571 sowohl einen Protronenpumpenhemmer als auch nicht steroidale Antirheumatika.

Über einen mittleren Zeitpunkt von fünf Jahren traten 3.229 gastrointestinale Blutungen auf. Die Inzidenzraten für Blutungen (Ereignisse pro 100 Patientenjahre) betrugen 1,8 für Patienten, die Protronenpumpenhemmer einnahmen, und 2,1 für $\mathrm{Pa}$ tienten, die keine Protronenpumpenhemmer einnahmen. Das Blutungsrisiko war damit um $28 \%$ reduziert und dieser Unterschied war statistisch signifikant. Dabei gab es keinen Unterschied, welcher Protronenpumpenhemmer und welches nicht steroidale Antirheumatikum verwendet wurden.

Schlussfolgerungen: Patienten, die nach einem Herzinfarkt mit antithrombotischer Therapie behandelt werden und gleichzeitig nicht sterodiale Antirheumatika einnehmen, profitieren von der gleichzeitigen Gabe von Protronenpumpenhemmern bezüglich gastrointestinaler Blutungen.

\section{- Kommentar von Hans-Christoph Diener, Essen}

\section{Patienten gut aufklären}

Hier handelt es sich nicht um eine randomisierte Studie, sondern um die Analyse eines großen Patientenregisters aus Dänemark. Die Ergebnisse wurden an Patienten mit Myokardinfarkt erhoben, lassen sich aber mit hoher Wahrscheinlichkeit auch auf Patienten übertragen, die nach einer TIA oder einem ischämischen Insult mit Thrombozytenfunktionshemmern behandelt werden. Die Ergebnisse legen nahe, dass Patienten die mit Thrombozytenfunktionshemmern behandelt werden und gleichzeitig beispielsweise wegen Rücken- oder Gelenkschmerzen nicht steroidale Antirheumatika einnehmen, Protronenpumpenhemmer erhalten sollten. Patienten müssen auch bei der Krankenhausentlassung darauf aufmerksam gemacht werden, dass die Einnahme von nicht steroidalen Antirheumatika, die weitgehend nicht verschreibungspflichtig sind, das Risiko gastrointestinaler Blutungen erhöhen kann.

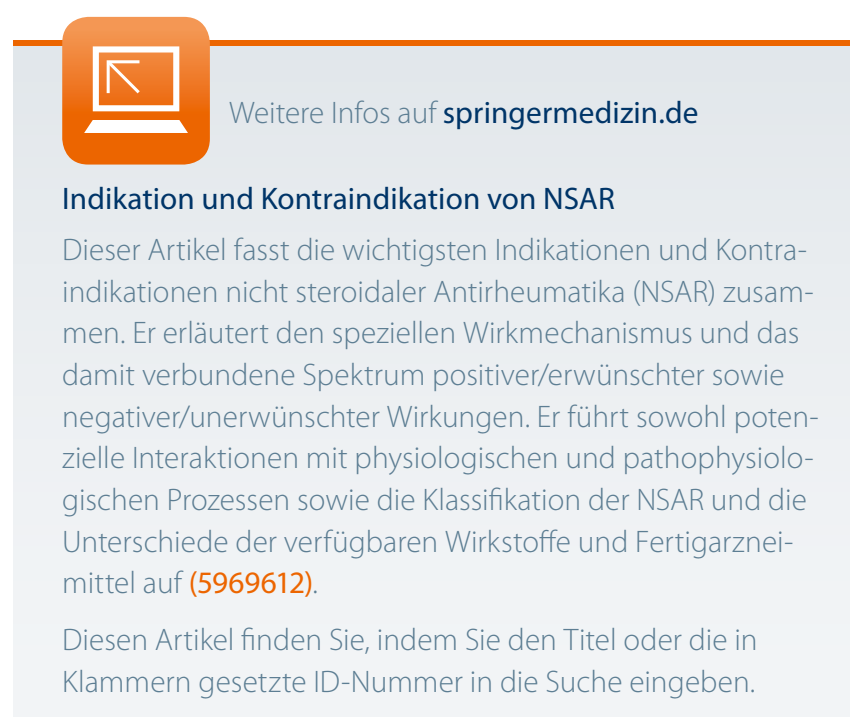

